Editorial

\title{
Die neue Redaktion stellt sich vor La nouvelle rédaction se présente
}

«Die relativ junge Existenz der modernen Naturwissenschaften, denen Medizin und Handwerk viel mehr zu Gevatter gestanden haben als die aus dem Altertum heraufgewachsene Philosophie, hat - neben Terminologie-Schwierigkeiten - es ganz natürlich mit sich gebracht, daß diese Gebiete von der eigentlichen Geschichtswissenschaft wenig gepflegt werden.

Solchem Mangel abzuhelfen und auch für die Schweiz über die Vortragstätigkeit unserer Gesellschaft hinaus ein kontinuierliches Kristallisations- und Vitalitätszentrum historischer Interessen innerhalb dieser Gebiete zu schaffen, ist die kleine Zeitschrift gedacht, die sich Conrad Gessner zum Patron wählt, ihn, den humanistischen Zürcher Arzt und Vater der Bibliographie, der in Basel promoviert und in Lausanne unter bernischer Oberhoheit zeitweise akademisch tätig gewesen.»

Diese Sätze stehen auf der 1. Seite des GESNERUS, Jahrgang 1 (1943/44). Der aus dem Elsaß gebürtige Zürcher Zoologe und Wissenschaftshistoriker Jean Strohl (1886-1942), dem unsere Zeitschrift seine Entstehung verdankt, hat sie kurz vor seinem Tod geschrieben. Der GESNERUS konnte trotz diesem Schicksalsschlag im folgenden Jahr erstmals erscheinen: der Pharmakologe Hans Fischer, zugleich als Historiker der Medizin schriftstellerisch tätig, übernahm die Redaktion und hatte sie bis zum 30. Jahrgang (1973) ununterbrochen inne. Fischer betont (loc. cit., S. 3 f.):

«Wenn etwas den besonderen Charakter dieser Zeitschrift ausmachen soll, so ist es die lebendige Beziehung zur Gegenwart: Medizin- und Naturwissenschaftsgeschichte sollen im GESNERUS nicht eine traditionell-antiquarische Angelegenheit werden, er soll nicht ein Sammel- und Raritätenkasten sein, sondern in erster Linie ein Ort, an welchem neben historischer Einzelforschung auch allgemeine, wissenschaftsmethodische und naturphilosophische Probleme erörtert werden können, eine kleine Stätte, an welcher durch Besinnung auf die ideellen Grundlagen von Naturwissenschaft und Medizin die Einheit der Forschung betont und der lebendige Zusammenhang im historischen Gang einer 
Entdeckung oder einer Idee aufgezeigt werden soll. So sieht GESNERUS seine Aufgabe auch darin, dem so notwendigen synthetischen Bestreben in den Naturwissenschaften und in der Medizin entgegenzukommen und durch Betonung des Erkenntniszusammenhanges zu einer Ideengeschichte der Naturwissenschaft und Medizin gelegentliche Bausteine zu liefern.»

Auf dieser Linie hat sich GESNERUS seither entwickelt, unter Fischer wie unter seinen Nachfolgern in der Redaktion: Hans H. Walser, Heinz Balmer und Carl Haffter. Das Programm hat sich mit der Zeit erweitert: Die Geschichte der Psychiatrie fand besondere Aufmerksamkeit, und immer mehr ist namentlich die Geschichte der Medizin auch als Sozialgeschichte zur Geltung gekommen. Diese Entwicklung geht Hand in Hand mit dem wechselnden Interesse der allgemeinen Geschichtswissenschaft an der Medizin, an ihren Vertretern und Institutionen sowie an ihrem Objekt, den kranken Menschen und ihren Krankheiten, besonders den epidemischen. (Man ist versucht zu sagen: die modernen Historiker widmen sich ebenso eifrig den Pestzügen wie die früheren den Kriegszügen ...)

Krankheiten, Heilkunde und Heilpersonen als integrierende Bestandteile einer weitgefaßten Allgemeingeschichte - diese Auffassung eröffnet große Perspektiven und fruchtbare Möglichkeiten gegenseitiger Bereicherung. Für die Geschichte der Naturwissenschaften gilt ähnliches; ein Zeugnis dafür, daß solch interdisziplinäre Möglichkeiten auch verwirklicht werden, stellt die 1988 erfolgte Schaffung eines gemeinsamen Lehrstuhles für Geschichte und Philosophie der Naturwissenschaften durch die beiden Fakultäten «Sciences» und «Lettres» an der Universität Genf dar (s. S. 124 dieses Heftes). GESNERUS stellt sich mit Überzeugung auch in den Dienst dieser interdisziplinären Forschungs- und Betrachtungsweise.

Die schöne Aufgabe, den GESNERUS in seiner Vielseitigkeit auf dem hohen Niveau zu halten, dem er sein Ansehen im In- und Ausland verdankt, wird nun von einer fünfköpfigen Redaktions-Equipe übernommen:

- Huldrych M.Koelbing, Dr.med., ist Chefredaktor. Von der Augenheilkunde wechselte er über einen wissenschaftlichen Redaktorenposten (Documenta Geigy) in die vollamtliche medizinhistorische Tätigkeit hinüber: 1965 an der Universität Basel habilitiert, leitete er 1971-1988 als Ordinarius das Medizinhistorische Institut der Universität Zürich. Seine Interessengebiete sind vor allem die Medizin der Antike und der Renaissance, die Geschichte der Augenheilkunde und die Pest. 
- Antoinette Stettler, Dr. med., ist ebenfalls für Geschichte der Medizin und der Pharmazie zuständig und redigiert die englischen Summaries. Nach langen Auslandaufenthalten lebt und praktiziert sie in Bern. Seit den sechziger Jahren arbeitet sie medizinhistorisch. Ihre Hauptgebiete: Seuchengeschichte und Geschichte medizinischer Begriffe und Konzepte.

- Philippe Mudry, Dr. ès lettres, représente, au sein de notre équipe, à la fois la Suisse romande et la philologie classique; par conséquent, sa collaboration revêt une double importance. Mudry est professeur de langue et littérature latines à l'Université de Lausanne. Il s'intéresse particulièrement à la médecine romaine.

- Hans Konrad Schmutz, Dr.phil., leitet die naturwissenschaftlichen Sammlungen der Stadt Winterthur und betreut in unserer Redaktion die Geschichte der Naturwissenschaften. Seiner Haupt-Studienrichtung nach ist er Anthropologe; die Geschichte der Anthropologie, namentlich im 18. Jh., beschäftigt ihn seit langem.

- Heidi Seger, Diplom-Bibliothekarin, steht der Bibliothek des Medizinhistorischen Instituts der Universität Zürich vor. Sie organisiert und überwacht die Bücher-Rezensionen - eine für die Orientierung auf unseren Fachgebieten unentbehrliche Sparte der Zeitschrift - und liest Korrekturen.

Zum Schluß möchte ich einen dreifachen, herzlichen Dank aussprechen:

- Herrn Prof. Dr. Carl Haffter, unserem souveränen Vorgänger, und Frau Dr.med. Ruth Haffter in Basel, die mir die Akten und Materialien der Redaktion wohlgeordnet übergeben haben.

- Meinen vier Redaktionskolleginnen und -kollegen, die sich ohne Zaudern und mit Freude für die neue Aufgabe zur Verfügung gestellt haben; ohne diese spontane und kräftige Unterstützung hätte ich die Verantwortung für unsere Zeitschrift nicht übernehmen können.

- Dem Zürcher Medizinhistorischen Institut, welches das Redaktionssekretariat besorgt.

Im Namen der gesamten Redaktion bitte ich die Leser des GESNERUS um ihr Wohlwollen. Wir nehmen gerne Ihre Anregungen und gegebenenfalls auch Thre Kritik entgegen und empfehlen uns Ihrer Aufmerksamkeit.

Huldrych M. Koelbing 